\title{
Neuronal Correlates of Cross-Modal Transfer in the Cerebellum and Pontine Nuclei
}

\author{
Matthew M. Campolattaro, ${ }^{1}$ Alireza Kashef, ${ }^{1}$ Inah Lee, ${ }^{2}$ and John H. Freeman ${ }^{1}$ \\ ${ }^{1}$ Department of Psychology, University of Iowa, Iowa City, Iowa 52242, and ${ }^{2}$ Department of Brain and Cognitive Sciences, Seoul National University, Seoul \\ 151-742, Korea
}

Cross-modal transfer occurs when learning established with a stimulus from one sensory modality facilitates subsequent learning with a new stimulus from a different sensory modality. The current study examined neuronal correlates of cross-modal transfer of pavlovian eyeblink conditioning in rats. Neuronal activity was recorded from tetrodes within the anterior interpositus nucleus (IPN) of the cerebellum and basilar pontine nucleus (PN) during different phases of training. After stimulus preexposure and unpaired training sessions with a tone conditioned stimulus (CS), light CS, and periorbital stimulation unconditioned stimulus (US), rats received associative training with one of the CSs and the US (CS1-US). Training then continued on the same day with the other CS to assess cross-modal transfer (CS2-US). The final training session included associative training with both CSs on separate trials to establish stronger crossmodal transfer (CS1/CS2). Neurons in the IPN and PN showed primarily unimodal responses during pretraining sessions. Learningrelated facilitation of activity correlated with the conditioned response (CR) developed in the IPN and PN during CS1-US training. Subsequent CS2-US training resulted in acquisition of CRs and learning-related neuronal activity in the IPN but substantially less little learning-related activity in the PN. Additional CS1/CS2 training increased CRs and learning-related activity in the IPN and PN during CS2-US trials. The findings suggest that cross-modal neuronal plasticity in the PN is driven by excitatory feedback from the IPN to the PN. Interacting plasticity mechanisms in the IPN and PN may underlie behavioral cross-modal transfer in eyeblink conditioning.

\section{Introduction}

The cerebellum and basilar pontine nuclei (PN) are necessary for acquisition and retention of eyeblink conditioning (Thompson and Steinmetz, 2009). The PN relay conditioned stimulus (CS) information to the cerebellum, which converges with unconditioned stimulus (US) input to form and store the memory underlying the eyeblink conditioned response (CR) (Steinmetz et al., 1989; Mauk and Donegan, 1997; Thompson and Steinmetz, 2009).

Neurons in the PN provide the necessary CS input to the cerebellum for eyeblink conditioning (Steinmetz et al., 1986, 1987, 1989; Mauk and Donegan, 1997; Hesslow et al., 1999; Thompson and Steinmetz, 2009; Halverson and Freeman, 2010a,b). Lesions of the PN or their axons in the middle cerebellar peduncle prevent CS information from reaching the cerebellum, which severely impairs eyeblink conditioning (Lewis et al., 1987; Steinmetz et al., 1987; Halverson and Freeman, 2010a,b). During conditioning neurons within the interpositus nucleus (IPN), and also the red nucleus, send feedback to the PN (McCormick et al., 1983; Cartford et al., 1997; Clark et al., 1997; Bao et al., 2000). This feedback may provide input that is necessary to induce learning-related changes in activity within the PN.

Received Aug. 8, 2010; revised Jan. 18, 2011; accepted Jan. 24, 2011.

This research was supported by National Institute for Mental Health Grants MH080005 (J.H.F.) and MH079971 (I.L.) and World Class University Program R32-10142 (I.L.).

Correspondence should be addressed to John H. Freeman, Department of Psychology, University of lowa, E11 Seashore Hall, lowa City, IA 52242. E-mail: john-freeman@uiowa.edu.

DOI:10.1523/JNEUROSCI.4142-10.2011

Copyright $\odot 2011$ the authors $\quad 0270-6474 / 11 / 314051-12 \$ 15.00 / 0$
Cross-modal learning is established by initially training with a CS of a particular sensory modality (e.g., auditory or visual, CS1) and subsequently switching the CS to a different sensory modality (CS2). Cross-modal transfer occurs when learning with CS2 develops at an accelerated rate relative to initial training with CS1 (Kehoe et al., 1984; Holt and Kehoe, 1985; Nahinsky et al., 2004; Campolattaro and Freeman, 2009). This type of learning results from the general transfer of the CS-US association, not primary stimulus generalization. General transfer occurs when responding is not immediately elicited by presentations of CS2, but acquisition is enhanced with additional training. Primary stimulus generalization occurs when responding to presentations of CS2 is immediate. Because plasticity in the cerebellum is necessary to establish and maintain the eyeblink CR memory, it was hypothesized that cerebellar plasticity is also important for establishing cross-modal transfer. Support for this hypothesis was found in a recent experiment that showed that unilateral inactivation of the cerebellum during training with either CS1 or CS2 completely blocked cross-modal transfer of eyeblink conditioning (Campolattaro and Freeman, 2009).

The present experiment was designed to examine neuronal activity in the IPN and PN during cross-modal transfer. The primary goal of this experiment was to examine multimodal (auditory and visual) neuronal activity in the IPN and PN before, during, and after cross-modal training. Multimodal neuronal responses that emerge during cross-modal training could play a causal role in behavioral cross-modal transfer. This experiment was also designed to determine whether neuronal cross-modal transfer in the IPN precedes cross-modal transfer in the PN, 
which would suggest that feedback from the IPN is responsible for driving neuronal activity changes in the PN during cross-modal training, as demonstrated previously for learning with a single CS (Clark et al., 1997).

\section{Materials and Methods}

Subjects. Subjects were 18 male Long-Evans rats $(200-250 \mathrm{~g}), \sim 150 \mathrm{~d}$ old at the beginning of the experiment. The rats were housed in Spence Laboratories of Psychology at the University of Iowa with a $12 \mathrm{~h}$ light/dark cycle, with light onset at 7:00 A.M. The procedures were approved by the University of Iowa Institutional Animal Care and Use Committee.

Surgery. Approximately 1 week before training, rats were removed from their home cage and anesthetized with isoflurane. After the onset of anesthesia, a craniotomy was performed above the left IPN [anterior-posterior (AP), $-11.5 \mathrm{~mm}$; media-lateral (ML), $+2.6 \mathrm{~mm}$ ], the right lateral PN (AP, $-7.3 \mathrm{~mm}$; ML, -1.5 $\mathrm{mm}$ ), or right medial PN (AP, $-7.4 \mathrm{~mm}$; ML, $-0.6 \mathrm{~mm}$ ) using a small trephine drill bit, and then the dura matter was carefully removed. The neural connectivity that corresponds to the conditioned eye is primarily the ipsilateral IPN and contralateral PN. A multielectrode recording device (i.e., hyperdrive) was then positioned to align the tetrode bundles with the openings in the skull. In some of the rats $(n=$
A

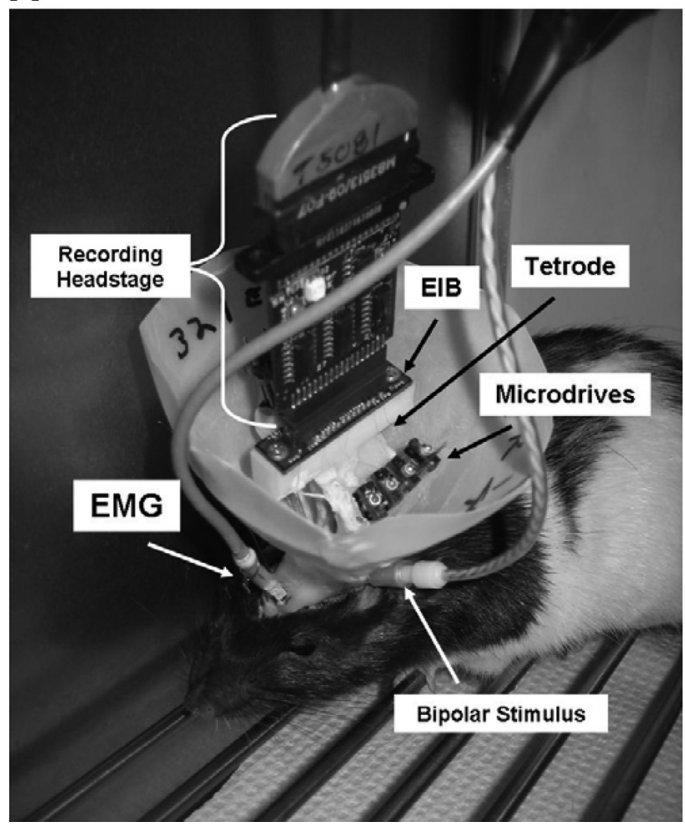

B
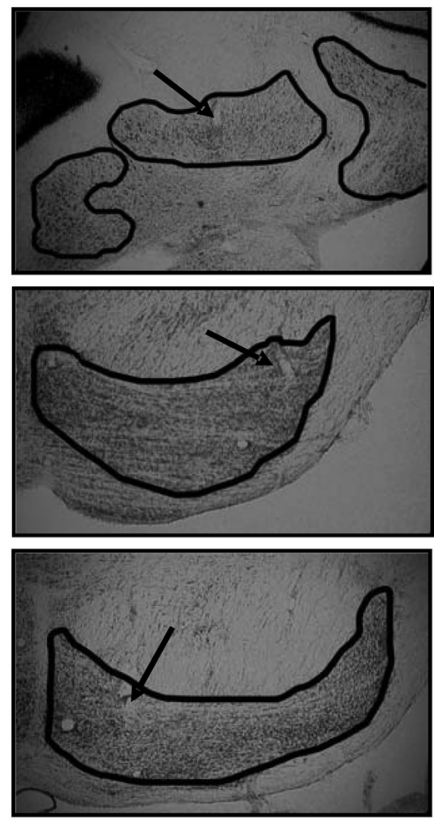

Figure 1. A, Image of a rat that depicts the eyeblink and neuronal recording equipment. Orbicularis oculi activity was recorded with EMG electrodes. The bipolar stimulus cable was used to deliver the periorbital stimulation unconditioned stimulus. Tetrodes were secured to the EIB and individually housed within microdrives. The recording head stage relayed unit activity from the EIB to the recording software. $\boldsymbol{B}$, Image showing locations of tetrode tips in the cerebellar anterior interpositus nucleus (top), lateral pontine nucleus (middle), and medial pontine nucleus (bottom).

12), two bundles of four tetrodes (three recording/one reference) were positioned above the IPN and PN [dorsal-ventral (DV), $-10.0 \mathrm{~mm}$ ]. In other rats, a bundle of seven tetrodes (six recording/one reference) was positioned above the IPN $(n=3)$. Low viscosity silicon (Kwik-Sil; World Precision Instruments) was used to seal the gap between the skull opening and the tetrode bundles. The hyperdrive assembly was then fixed to the skull with bone cement (Zimmer) and was grounded to a silver wire that was attached to a stainless steel screw fixed to the right frontal bone of the skull. Recording tetrodes were then lowered to $0.5 \mathrm{~mm}$ above their target (IPN DV, $5.0 \mathrm{~mm}$; PN DV, $9.5 \mathrm{~mm}$ ). Reference tetrodes were also lowered above each target (IPN DV, $2.0 \mathrm{~mm}$; PN DV, $5.0 \mathrm{~mm}$ ). The rats were then fitted with differential electromyograph (EMG) electrodes that were placed in the left upper eyelid muscle (orbicularis oculi). The EMG ground electrode was attached to a stainless steel skull screw. The EMG leads terminated in gold pins held in a plastic connector, which was secured to the skull with bone cement. A bipolar stimulating electrode (for delivering the US) was implanted subdermally, immediately caudal to the left eye that was secured to the skull with bone cement.

Tetrode drives. A custom-manufactured tetrode drive assembly with a 27-channel electronic interface board (EIB-27; Neuralynx) was used in this experiment (Fig. 1A). Tetrodes were loaded into moveable microdrives that were connected to the drive assembly. Each tetrode consisted of four insulated nichrome microwires ( $12 \mu \mathrm{m}$ diameter; Kanthal) that were twisted and partially fused together by melting the insulation with heat. Each microwire in a tetrode was secured to an individual channel in the EIB with a small gold pin, except for tetrodes designated as reference electrodes that had all four microwires secured into a single channel. After a tetrode was loaded into a microdrive, the tip was lowered into a gold solution (Sifco Process) in which a small amount of current $(\sim 0.1$ $\mu \mathrm{A}$ ) was passed through it to adjust impedance to $350-1000 \mathrm{k} \Omega$ (Impedance Tester IMP-1; Bak Electronics).

Conditioning apparatus. The conditioning apparatus consisted of a smallanimal sound attenuation chamber (BRS/LVE). Within the sound attenuation chamber was a small-animal operant chamber (BRS/LVE) in which a rat was kept during conditioning. One wall of the operant chamber was fitted with two speakers. The back wall of the sound attenuation chamber was equipped with a small house light and an exhaust fan. A light bulb (for delivering the light CS) was located on the back wall of the sound attenuation chamber, positioned directly behind the operant chamber. The electrode leads from the rat's head stage were fed through a motorized commutator that connected to peripheral equipment and a desktop computer. Computer software controlled the delivery of stimuli and the recording of eyelid EMG activity (JSA Designs). The US (1-2 mA, direct constant current) was delivered through a stimulus isolator (model number 365A; World Precision Instruments). EMG activity was recorded differentially, filtered (500-5000 $\mathrm{Hz}$ ), and integrated (JSA Designs and Neuralynx).

Behavioral training procedures. The experimental design is shown in Table 1. All rats recovered from surgery for 1 week before beginning training. The CSs were a $2 \mathrm{kHz}$ tone and a $1.65 \mathrm{~W}$ light (order counterbalanced), and the US was a 1-2.0 $\mathrm{mA}$ periorbital shock. The duration of each CS was $500 \mathrm{~ms}$, in which the offset of the $25 \mathrm{~ms}$ US coincided with the offset of the CS. In phase 1 (preexposure training), each rat received one session of 50 tone-alone trials $(500 \mathrm{~ms})$ and 50 light-alone trials (500 $\mathrm{ms}$ ) with an intertrial interval (ITI) that averaged $30 \mathrm{~s}$ (range, 15-45 s). The preexposure phase was used to assess sensory-related responses in the IPN and PN. In phase 2 (unpaired training), each rat received 50 unpaired presentations each of the tone, light, and shock unconditioned stimulus (25 ms) with an ITI that averaged $15 \mathrm{~s}$ (range, 5-25 s). The unpaired phase was used to assess sensory-related responses in the presence of the shock stimulus. In phase 3 (CS1-US acquisition), rats were given 100-trial sessions of paired training with the first CS (CS1, either the tone or light) and the US with an average ITI of $30 \mathrm{~s}$ (range, 15-45 s). Every 10th trial of paired training was a CS-alone probe trial (10 probe trials per 100-trial session). Up to three additional training sessions were necessary for some rats $(n=6)$ that did not obtain a CR response rate of $60 \%$ by session 5 . Four rats reached this criterion on session 6 and two on session 8. Phase 4 (crossmodal transfer) occurred on the same day as the last session of CS1 acquisition. In this phase, all rats received a 100-trial session of paired training with the second CS (CS2), which differed from CS1 in sensory modality, and the US (CS2-US). It was necessary that the last session of acquisition to CS1 and conditioning with CS2 occurred on the same day to record from the same neurons during cross-modal transfer. In phase 5 (CS1/CS2 training), all rats received a 100-trial session of intermixed trials with 50 paired presentations 
Table 1. Experimental design

\begin{tabular}{lllll}
\hline $\begin{array}{l}\text { Phase 1: } \\
\text { preexposure }\end{array}$ & $\begin{array}{l}\text { Phase 2: } \\
\text { unpaired }\end{array}$ & $\begin{array}{l}\text { Phase 3: } \\
\text { CS1 paired }\end{array}$ & $\begin{array}{l}\text { Phase 4: } \\
\text { cross-modal }\end{array}$ & $\begin{array}{l}\text { Phase 5: } \\
\text { CS1/CS2 paired }\end{array}$ \\
\hline Tone & Tone - & CS1+ & CS2 + & CS1+ \\
Light & Light - & & & CS2 + \\
& + & & &
\end{tabular}

Design summary: Tone, $500 \mathrm{~ms}, 2$ kHz CS; Light, $500 \mathrm{~ms}, 1.25 \mathrm{WCS} ;+$, a $25 \mathrm{~ms}$ US was presented; - , no US. CS1 and CS2 were the tone and light CSs (counterbalanced).

A Pre-Exposure

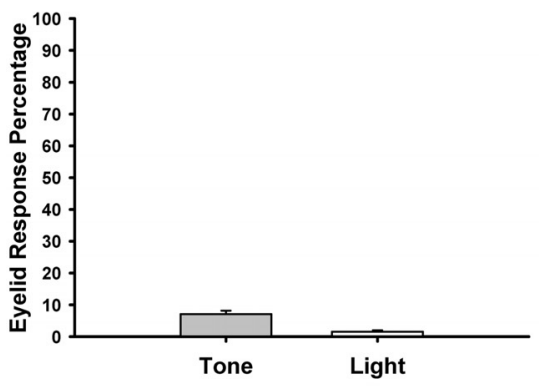

\section{B Unpaired}

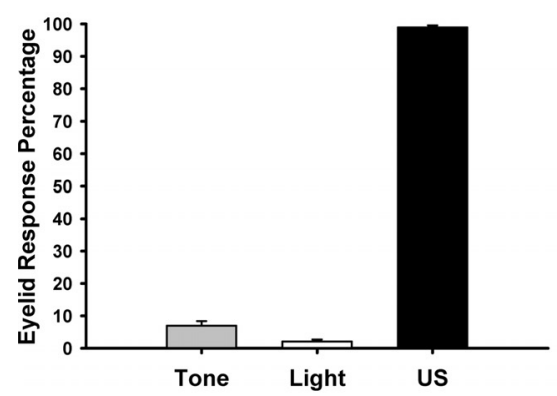

C Paired (session)

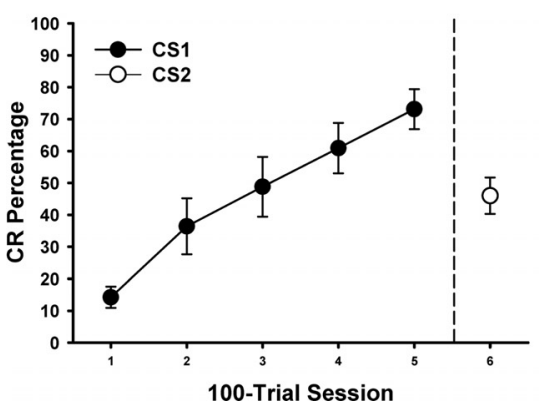

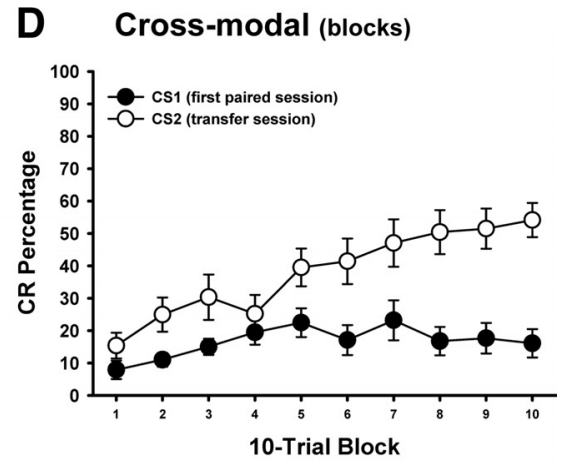

E

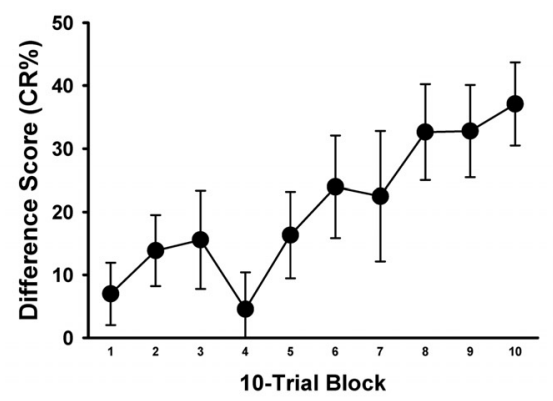

F CS1/CS2 Paired

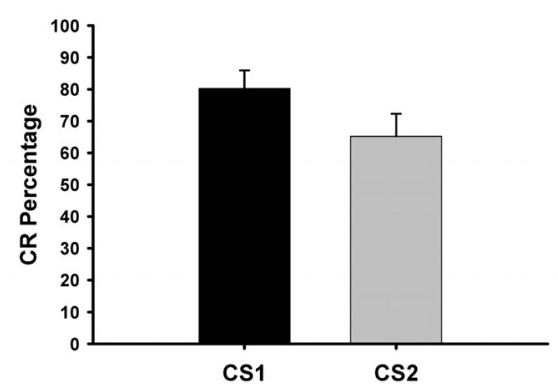

Figure 2. Mean \pm SEM eyeblink response percentage during preexposure $(\boldsymbol{A})$ and unpaired training $(\boldsymbol{B})$ to the tone CS (gray bar), light CS (white bar), and shock US (black bar). C, Mean \pm SEM percentage of CRs during paired training with CS1 (black circles, sessions 1-5) and cross-modal transfer with CS2 (white circle, session 6). D, Mean \pm SEM percentage of CRs in 10-trial blocks for CS2 (white circles) during the transfer session and for CS1 (black circles) during the first paired session. $\boldsymbol{E}$, Mean \pm SEM percentage CRs in 10-trial blocks of within-subject differences between CS2 during the transfer session and CS1 during the first paired session. $\boldsymbol{F}$, Mean ( \pm SEM) percentage of CRs to CS1 (gray bar) and CS2 (white bar) during CS1/CS2 paired training.

of the tone and light CSs and the US. The purpose of this session was to evaluate behavioral and neuronal responses to both CS modalities after cross-modal transfer was well established.

Neuronal recording apparatus and unit sorting procedures. The activity of each channel was passed through a head-stage unity gain preamplifier (Fig. 1A) (Neuralynx). The output from the preamplifier was passed through a motorized commutator, fed into eight-channel programmable amplifiers (Neuralynx), filtered between 600 and $6000 \mathrm{~Hz}$, and amplified at a gain of 10,000-20,000. The outputs of the amplifiers were digitized and stored at $32 \mathrm{kHz}$ per channel in a computer-controlled acquisition system (Neuralynx). An automatic cluster cutting program (KlustaKwik; K. Harris, Rutgers University, Newark, NJ) was used to first identify and merge clusters with similar waveform properties using parameter such as peak, valley, and energy. These steps were used to isolate single nits from multiunit recordings. Clusters were then exported to an in Minnesota, Minneapolis, MN) to manually inspect and further refine clusters to ensure that they contained the spikes of only a single neuron.

Neuronal recording analyses. Peristimulus time histograms of unit activity in the IPN and PN were generated for the tone CS, light CS, and shock US that were used during phases 1 and 2. The duration of the CSs was divided into four time periods ( $125 \mathrm{~ms}$ ) that each contained 1012.5 -ms bins. The duration of the US was divided into two equal time periods (each 125 ms starting after US onset) that each contained 1012.5 -ms bins. Wilcoxon's signed rank tests were used to statistically compare the firing rates to the different stimuli relative to the pre-CS baseline. The Wilcoxon's signed rank test detects significant differences at approximately the same levels as $z$-score analysis without assumptions about the normality of the distribution of sample data (Freeman and Nicholson, 2000; Nicholson and Freeman, 2000, 2002, 2003; Freeman and Muckler, 2003; Halverson et al., 2010). Significant differences were determined at the $p<0.05$ value. This test compared each time period of the stimulus to an equal baseline period (125 ms) that immediately preceded the onset of the stimulus. The same tests were used to detect changes in firing rate to the CSs during paired training in the subsequent training phases, except that the CSs were divided into four $112.5 \mathrm{~ms}$ blocks that each contained 10 11.25-ms bins. CS durations for paired training trials were adjusted to reflect the interstimulus interval between the CS and US. Separate Wilcoxon's signed rank tests were performed for trials when a CR was produced and when a CR was not produced to assess the development of learning-related activity. $\chi^{2}$ tests were used to make statistical comparisons between the percentages of units responding in different ways during paired and unpaired training.

Tetrode placement and histology. After completion of the experiment, small marking lesions were made by passing $10 \mu \mathrm{A}$ of direct current for $10 \mathrm{~s}$ through one channel of each tetrode. Twenty-four hours later, the rat was deeply anesthetized with an overdose of sodium pentobarbital $(90 \mathrm{mg} / \mathrm{kg})$ and transcardially perfused with $0.9 \%$ PBS, followed by $3 \%$ buffered formalin. The brains were removed from the skull, postfixed in $30 \%$ sucrose-formalin, and subsequently sectioned at $50 \mu \mathrm{m}$ on a sliding microtome (American Optical). All sections were mounted on slides, stained with thionin, and examined for tetrode placement.

\section{Results}

Tetrode placement

A total of 558 unit recordings were obtained from tetrodes that were located within the IPN (249) and PN (309). Within the PN, 201 units were located in the lateral region and 108 units were 


\section{Unpaired Training}

\section{Interpositus}

Tone

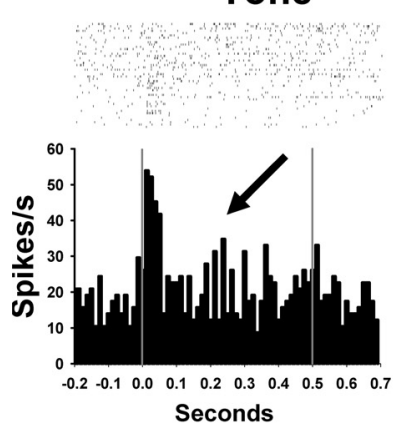

Unimodal Tone

Tone

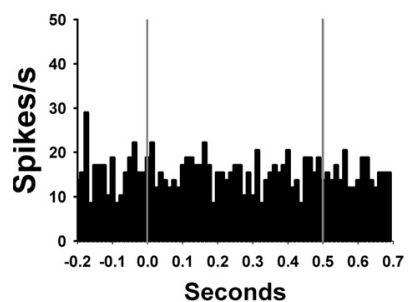

Unimodal Light

Tone

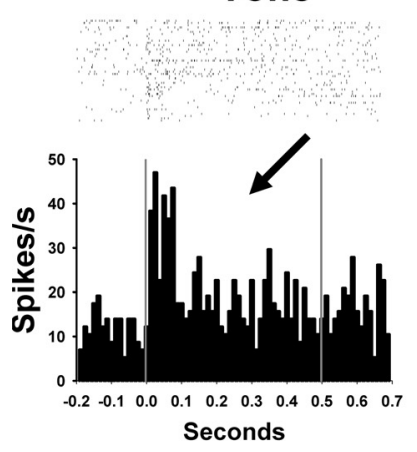

Multimodal
Light

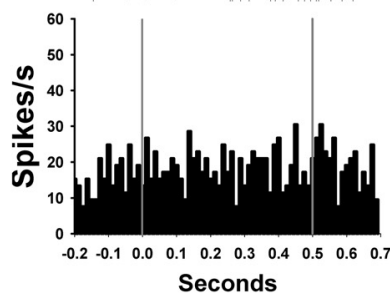

Light

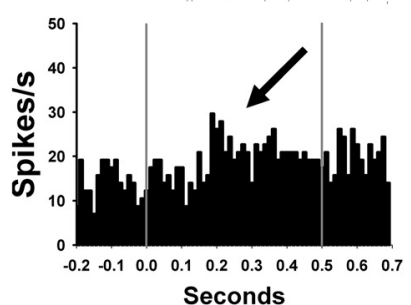

Light

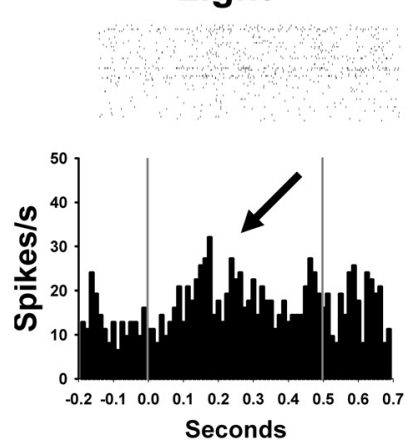

Seconds
Tone

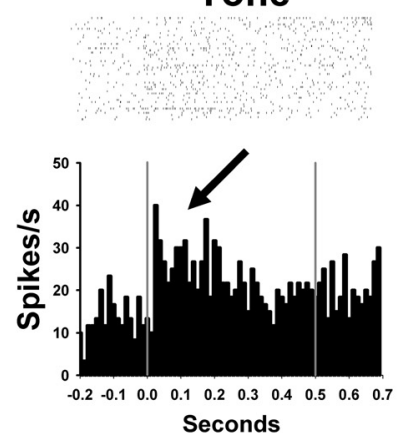

Light

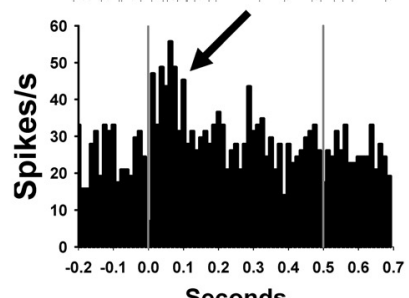

Tone

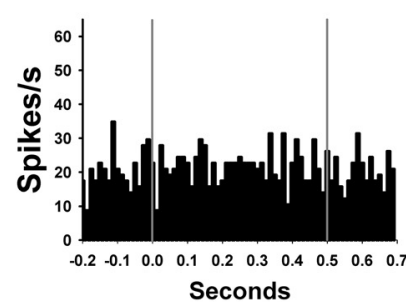

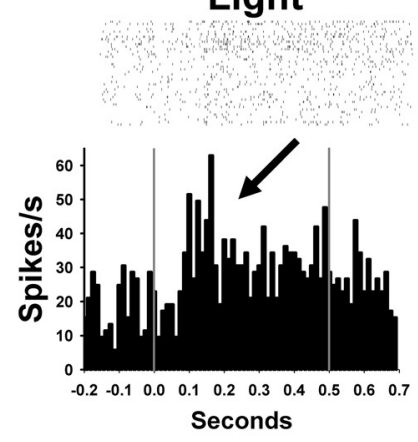

Light

Light

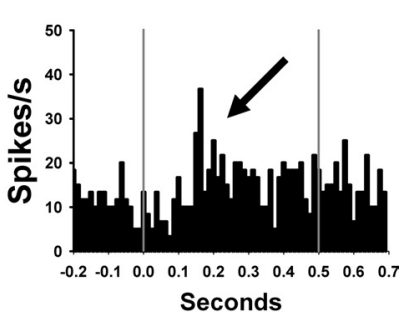

Figure 3. Histograms and raster plots of single-unit activity in the anterior interpositus nucleus (left) and pontine nucleus (right) during presentations of the tone and light CSs in unpaired training. The unimodal tone units (top) show a large increase in activity immediately after the onset of the tone CS but no response to the light CS. The unimodal light units (middle) show a large increase in activity after the onset of the light CS but no response to the tone CS. The multimodal units (bottom) show a large increase in activity during both the tone and light CSs. Arrows point to the peak of stimulus-elicited activity. Each row contains activity from an individual unit. The gray line represents the onset and offset of each CS.

located in the medial region. Figure $1 B$ presents examples of marking lesions that show the locations of the tips of tetrodes in the IPN, lateral PN, and medial PN, respectively. Reference tetrodes for the IPN were typically located within the white matter region beneath the cerebellar cortex, directly dorsal to the IPN. Reference tetrodes for the PN were typically located in the reticular pons. To have a reference electrode that was suitable for recording, it was necessary to adjust them to an area with minimal neuronal activity.
Behavioral data

The training conditions that were used in this experiment (Table 1) were effective for producing cross-modal transfer (i.e., rapid acquisition with CS2) of eyeblink conditioning. Eyelid closure percentages to the tone and light CSs during the preexposure and unpaired training sessions were consistently low (7.1 and 1.6\%, respectively) (Fig. $2 A, B$ ). In contrast, the percentage of eyelid responses elicited by the US was consistently high during the unpaired training session (99.0\%) (Fig. 2 B). During paired train- 
ing, rats acquired a high percentage of CRs to CS1 (tone or light) by their last session of training (76.5\%). During the CS1-to-CS2 transfer phase of training, CR percentage to CS2 was more than double the percentage obtained during the first session of paired training with CS1 (43.3 vs 16.6\%) (Fig. 2C). CR percentages from the transfer session were further inspected using 10-trial blocks and were compared with acquisition with CS1 during the first session of paired training. The percentage of CRs to CS2 was low on the first block of training (15.4\%) but steadily increased during the subsequent blocks to $54.2 \%$ by the 10 th block (Fig. $2 D$ ). In comparison, CR percentage to the first CS1 was consistently low on each of the 10-trial blocks of paired training during session $1(7.9-16.1 \%)$ (Fig. $2 D)$. These findings indicate that acquisition of CRs to the CS2 was facilitated relative to training with the CS1, and this facilitation was not attributable to primary stimulus generalization.

No statistical differences were found for CS modality (light vs tone) in this experiment, and analyses were, therefore, collapsed across the stimulus modality factor. A within-subjects repeatedmeasures ANOVA revealed a significant main effect of training session during paired training with $\operatorname{CS} 1\left(F_{(4,64)}=43.14, p<\right.$ 0.001). This effect was attributable to a steady increase in $C R$ percentage as a function of training (Fig. 2C). Analysis of the block data from the cross-modal transfer session with CS2 and the first session of paired training with CS1 revealed a significant interaction between CS order and training blocks $\left(F_{(9,144)}=3.19\right.$, $p<0.01$ ). A follow-up test (honestly significant difference test) showed that this interaction was attributable to faster acquisition to CS2 than CS1 on blocks 2, 3, and 5-10 ( $p<0.05)$ (Fig. 2D). Within-subject difference scores were calculated for each rat by subtracting the CR percentage obtained from the 10-trial blocks of CS1 (session 1) from the corresponding 10-trial blocks of CS2 (Fig. 2E). An ANOVA revealed that the difference scores calculated between CS1 and CS2 were greater than zero $\left(F_{(9,144)}=\right.$ $3.19, p<0.01)$. Analysis of CR percentage data obtained from CS1/CS2 training revealed that significantly more CRs were elicited by CS1 than CS2 $\left(t_{(16)}=2.95, p<0.05\right)$ (Fig. $\left.2 F\right)$. This finding shows that the magnitude of conditioning to CS1 was greater than the magnitude of conditioning to CS2 during the CS1/CS2 session, and, unlike CS1, conditioning to CS2 was still being acquired.

\section{Cerebellar and pontine neuronal activity during preexposure training}

Neurons recorded from the IPN and PN had average baseline (spontaneous) firing rates of 26.2 and $18.3 \mathrm{~Hz}$, respectively. During preexposure training, each rat received presentations of the tone and light CSs to assess sensory-related responses in the IPN and PN before learning. Neurons in the IPN and PN showed changes in activity during presentations of the tone and light CSs in preexposure training. The largest proportion of the responsive ( significantly different from baseline activity) neurons in the IPN showed changes in activity to one of the two stimuli, i.e., they were unimodal (tone, 13.9\%; light, 20.8\%). However, multimodal (tone and light) responses were also observed in the IPN during preexposure training, although less frequently than unimodal responses (7\%). PN neurons showed approximately equal proportions of unimodal (tone, 13.4\%; light, 26.8\%) and multimodal $(23 \%)$ neurons during preexposure training. Figure $4 \mathrm{~A}$ shows the percentage of units in the IPN (left) and PN (right) that exhibited significant increases or decreases for each CS period during preexposure training. Overall, more excitatory responses were elicited by the CSs in the PN relative to the IPN. Excitatory
A Pre-Exposure Training Interpositus
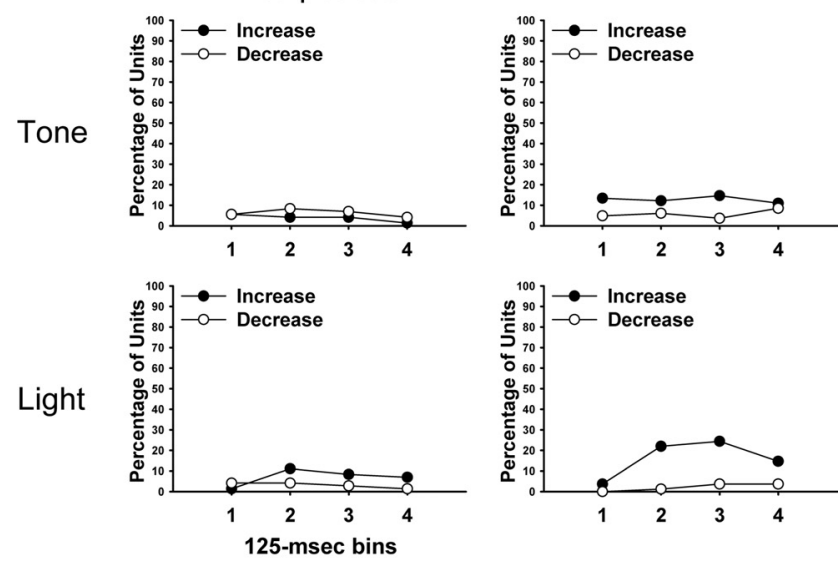

\section{B Unpaired Training}
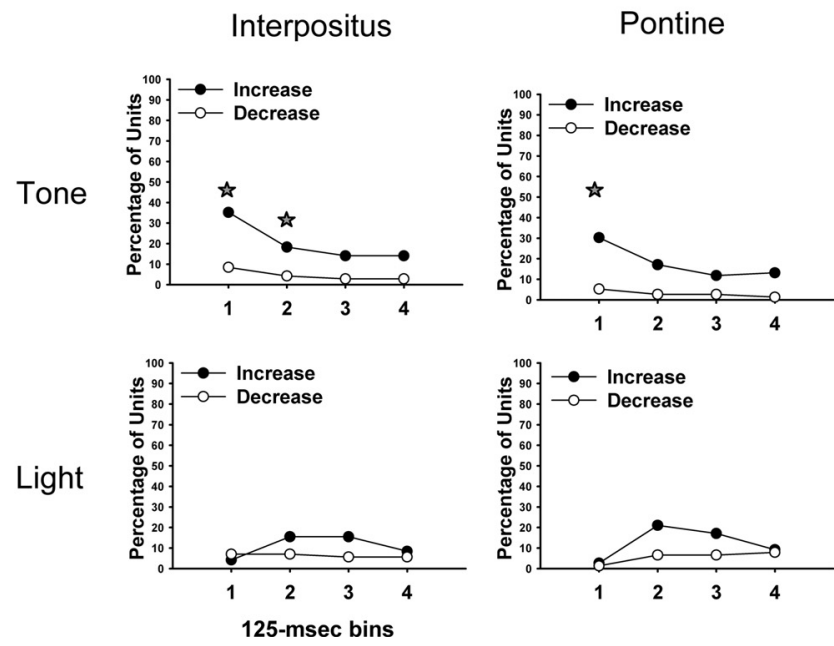

Figure 4. Percentage of units in the interpositus and pontine nucleus that exhibited significant increases (black circles) or decreases (white circles) in activity for each $125 \mathrm{~ms}$ bin (1-4) of the conditioned stimuli (tone or light) during preexposure $(\boldsymbol{A})$ and unpaired training $(\boldsymbol{B})$. A five-point star indicates a significant difference in bin percentages between preexposure and unpaired training.

responses to the tone CS in the $\mathrm{PN}$ were generally elicited at the beginning of the CS, whereas excitatory responses to the light CS typically occurred after $100 \mathrm{~ms}$. No significant differences were observed for inhibitory responses in the IPN and PN during this phase. The preexposure training results indicate that the majority of IPN neuronal responses to the CSs before learning were unimodal, whereas the PN neuronal responses included approximately equal proportions of unimodal and multimodal responses.

\section{Cerebellar and pontine neuronal activity during unpaired training}

Unpaired training consisted of unpaired presentations of the tone CS, light CS, and shock US to assess sensory-related responses in the presence of arousal produced by the aversive US. Similar to the preexposure session, multimodal and unimodal responses were observed from IPN and PN units during unpaired training. Examples of unimodal (tone or light responsive) and multimodal (tone and light responsive) single-neuron activity are shown in Figure 3. A higher percentage of responsive neurons 
were unimodal (IPN, 47.8\%; PN, 42.1\%) than multimodal (IPN, 26.8\%; PN, 26.3\%) during unpaired training.

Figure $4 B$ shows the percentage of units in the IPN and PN that exhibited significant increases or decreases for each CS bin during unpaired training. A general finding was that excitatory responses to the tone CS were more frequent in the IPN and PN during unpaired training with the US compared with the preexposure training without the US (Fig. 4, $A$ vs $B)$. No significant differences were observed for inhibitory responses in the IPN or PN during this phase.

Neuronal firing rates were also obtained after presentations of the US during this phase and analyzed with the same statistical procedures used for the CSs, except that baseline activity was compared with two US time intervals (each $125 \mathrm{~ms}$ ). Increased activity was commonly observed to the US in both the IPN (64.8\%) and $\mathrm{PN}(48.7 \%)$. Of those responsive units, the majority of activity was sustained during both time intervals for both the IPN (71.7\%) and PN (62.2\%).

The results from the unpaired training sessions indicate that neurons in the IPN and PN were primarily unimodal before training. There was a small population of multimodal neurons in both areas as well. Neuronal responses to the tone stimulus were generally enhanced during unpaired training relative to preexposure training. This effect is probably related to the arousing effects of the shock US. It is not clear why responses to the light stimulus were not also enhanced during unpaired training. Comparison of neuronal activity from the unpaired and CS-US paired training provides information about which changes in neuronal activity are attributable to associative learning. A major issue for this study was whether there would be a shift from primarily unimodal activity to multimodal activity in the IPN and $\mathrm{PN}$ as a result of cross-modal transfer, i.e., training with CS1-US pairings followed by training with CS2-US pairings.

\section{Cerebellar and pontine neuronal activity during initial cross-modal training}

The last session of CS1-US acquisition and the first session of CS2-US training occurred on the same day to record from the same neurons during initial cross-modal transfer. Examples of IPN and PN single neuron activity during CS1-US and CS2-US training are shown in Figure 5. Paired training with CS1 produced significant increases in activity in the IPN and PN in which a majority of the increase took place late in the CS period, which was the time when the CR was produced, a finding that replicates several previous studies (Freeman and Nicholson, 1999; Nicholson and Freeman, 2002; Halverson et al., 2010). As in previous onset of the US.

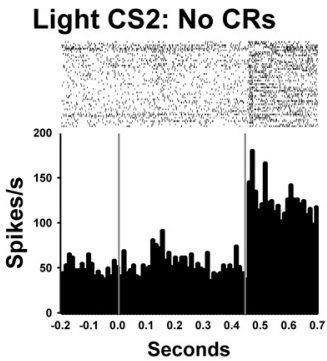

Tone CS2: No CRs

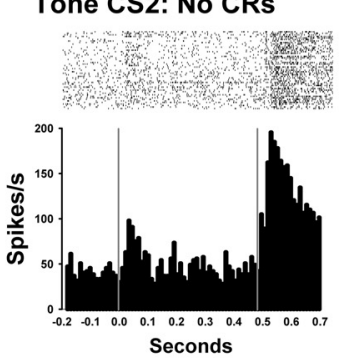

Light CS2: No CRs

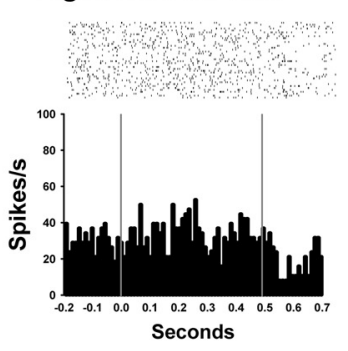

Tone CS2: No CRs

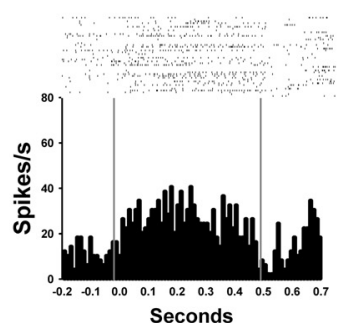

Seconds

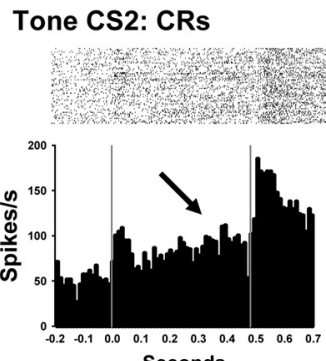

Seconds

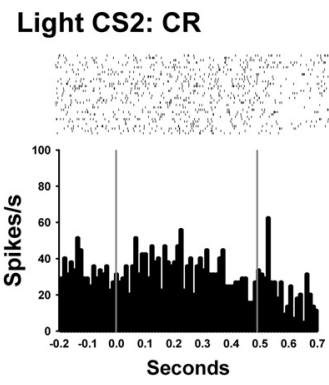

Tone CS2: CRs

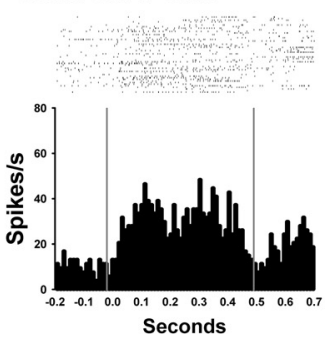

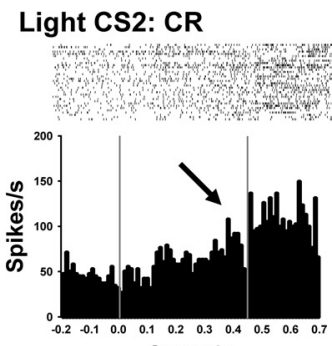

Figure 5. Histograms and raster plots of the activity of single neurons recorded from the anterior interpositus nucleus (top) and pontine nucleus (bottom) during initial cross-modal training. Rats were first given training with a light or tone conditioned stimulus (CS1-US) immediately followed by training with the other stimulus as the CS2 (CS2-US). All of the neurons showed is CR-related activity during CS2-US training as well. In contrast, the pontine neurons showed sensory responses to the CS2 but

studies, increases in IPN activity that occurred near the end of the CS1 were seen primarily on trials in which a CR was produced and preceded onset of the CR within trials. A generally accepted interpretation of this pattern of neuronal activity in the IPN has been that IPN neurons drive production of the CR by activating premotor neurons (Thompson and Steinmetz, 2009). IPN and PN neurons showed increased activity during the CS2 as well but it was weaker than seen with the CS1 (Fig. 5). Moreover, neurons in the PN showed less CR-related (greater activity on trials with a $\mathrm{CR}$ relative to trials without a CR) activity during the CS2 than neurons in the IPN (Fig. 5). The reduction in IPN and PN activity from CS1 to CS2 is most likely related to the development of learning during CS2-US training. That is, when neuronal activity 


\section{A Interpositus}

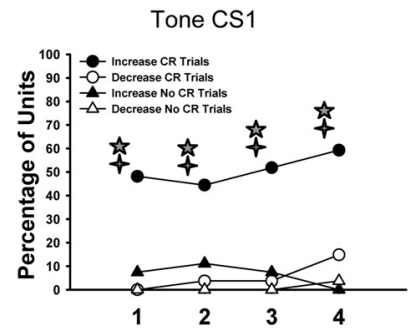

Light CS1

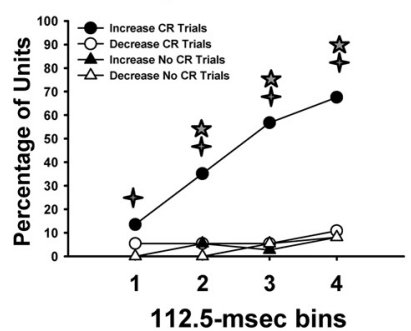

\section{B Pontine}

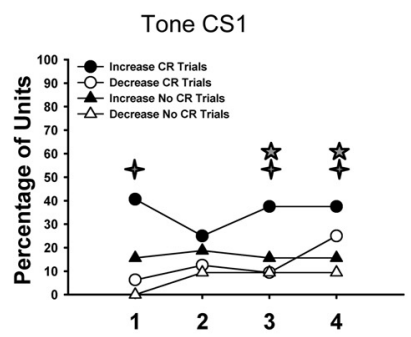

Light CS1
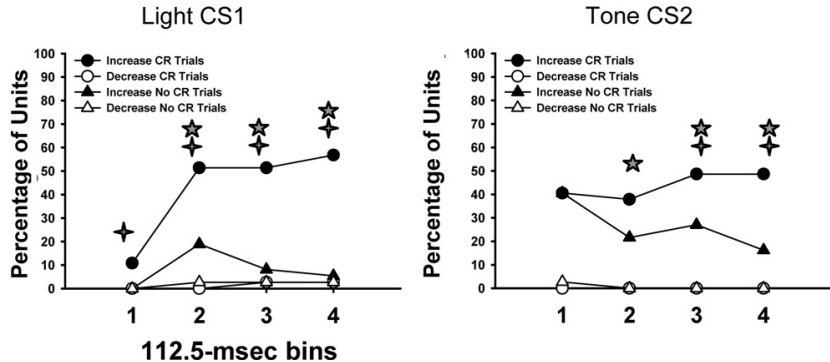

Figure 6. Percentage of units in the anterior interpositus nucleus $(\boldsymbol{A})$ and pontine nucleus (B) that exhibited significant increases (black plots) or decreases (white plots) in activity for each 112.5 ms bin (1-4) of the conditioned stimuli (CS1 and CS2) during initial cross-modal training, separated into CR (circles) and non-CR (triangles) trials. Four-point stars indicate significant differences in bin percentage between the $C R$ and no $C R$ trial types. Five-point stars indicate significant difference in bin percentage between paired and unpaired training (see Fig. $4 B) . \chi^{2}$ tests were used to make statistical comparisons between the percentages of units responding in different ways.

is pooled across all of the training trials for CS2, the initial trials with no learning decrease the average activity profiles. (An analysis of changes in IPN and PN activity within CS2-US training is presented below.) These data also suggest that neurons in the PN take longer to develop learning-related changes in activity than neurons in the IPN.

A breakdown of the general neuronal response patterns for tone-to-light and light-to-tone transfer is shown in Figure 6. Multimodal and unimodal responses were observed in the IPN and PN during the cross-modal transfer session. Multimodal activity was much more prevalent than unimodal activity during CS2-US training (Fig. 6, right column). Most of the neurons that responded during CS2-US training were multimodal. That is, this population of neurons responded to the CS1 and then also responded to the CS2. Overall, there were substantially more IPN neurons that showed increased activity during the CS2-US trials relative to the PN neurons (Fig. 6).

Figure 7 shows how IPN and PN neurons with different activity profiles during CS1-US training changed during CS2-US training. Neurons were classified as being response related (increased activity on trials with a CR), sensory related (increased responding not related to production of CRs), or showing no change in activity to presentations of a CS. A large proportion of the IPN neurons that showed changes in activity during CS2-US training were differentially active during trials with CRs during CS1-US training. Very few IPN neurons switched from no response or a sensory response during CS1-US training to a CRrelated response during CS2-US training. A smaller proportion of PN neurons showed CR-related changes in activity during CS2-US training relative to the IPN. Moreover, many of the PN neurons that showed greater activity during trials with a $\mathrm{CR}$ (response-related neurons) during CS1-US training showed nondifferential sensory-like responses during CS2-US training (Fig. 7).

No apparent segregation of stimulus modality was observed between regions in the medial and lateral PN. Tetrodes in those locations produced a wide variety of sensory and CR-modulated responses to both the light and tone CSs. Moreover, equal proportions of sensory and CR-related responses were found for the light and tone CSs in both the lateral and medial PN.

Learning-related changes in neuronal activity were seen in the IPN and PN as rats were switched from CS1-US training to CS2-US training. A substantial increase in IPN activity that corresponded with production of the $\mathrm{CR}$ was observed during CS2-US training. A higher percentage of neurons in the $\mathrm{PN}$ also showed CR-related activity during CS2-US training but still lower than the percentage of neurons showing CR-related activity in the IPN. The results suggest that the IPN acquired learningrelated plasticity earlier than the $\mathrm{PN}$ during this initial phase of CS2 acquisition. Neuronal activity was subsequently examined again when behavioral responding to the CS2 increased to levels more comparable with the CS1 during CS1/CS2 training to see whether PN neurons would "catch up" to IPN neurons in terms of learning-related activity (see below, Cerebellar and pontine neuronal activity during CS1/CS2 training).

\section{Cerebellar and pontine neuronal activity during the development of cross-modal transfer}

As mentioned above, pooling the data from all of the CS2-US trials may have partially obscured the development of learning-related activity in the IPN and PN during initial cross-modal transfer. To examine how unit activity changed during acquisition of CRs to CS2, the transfer session was divided into two parts based on a behavioral criterion. For each rat, performance during the cross-modal transfer session was divided into time periods before and after transfer occurred. The trials after a rat produced $40 \%$ CRs were in the posttransfer period, whereas trials before the $40 \%$ criterion were in the pretransfer period.

Figure $8 A$ shows examples of activity recorded from individual IPN and PN neurons during cross-modal training. Strong responding to CS1 was observed in the later periods of CS1, around the time when the CR typically occurred, for the IPN and PN neurons. When cross-modal training began with CS2, the IPN and PN neurons showed less of activity toward the end of 
CS2 relative to CS1 (Fig. $8 A$, before). As behavioral cross-modal transfer to CS2 developed, the IPN neuron showed a partial resurgence in activity toward the end of the CS2 (Fig. $8 A$, after). In contrast, the $\mathrm{PN}$ neuron failed to show evidence of activity toward the end of CS2. These neurons are illustrative of the general trends in the IPN and PN populations. Figure $8 B$ shows the percentage of units in the IPN that showed significant changes (excitatory or inhibitory) in activity during CS-US trials, including both CR and no CR trials, before and after behavioral transfer to CS2. A significantly greater percentage of IPN neurons showed increased activity (CR-related and sensory) during the CS2 after behavioral transfer occurred. This trend was observed when CS2 was either the tone or light CS (Fig. $8 B$ ). In contrast, $\mathrm{PN}$ neurons did not show a significant increase in activity during CS2 after behavioral transfer occurred (Fig. 8B). The absence of a difference in responding in the "before" and "after" time periods of cross-modal transfer in the PN suggests that learning-related changes in activity in the PN lagged behind the development of learning-related activity in the IPN.

\section{Cerebellar and pontine neuronal activity during CS1/CS2 training}

After the initial cross-modal transfer session, training continued the following day with intermixed CS1-US and CS2-US trials to determine whether more substantial behavioral and neuronal transfer to CS2 could be established, particularly in the PN, in which little neuronal transfer was seen during the initial session of CS2-US training.

The percentage of trials with CRs was higher on CS2-US trials during CS1/CS2 training relative to the initial cross-modal session. This increase in CR percentage corresponded with an increase in learning-related neuronal activity in the IPN and PN. Most of the IPN neurons (88.1\%) showed excitatory responses to both the tone and light CSs during training (Figs. 9-11). Many of the IPN neurons showed elevated activity toward the end of the CS period (CR-related and sensory) for both stimuli, and the IPN activity during CS2 was stronger than that seen in the initial cross-modal session (Fig. 9). PN neurons also showed more activity during CS2 trials (Fig. 9), with $48 \%$ showing CR-related and sensory multimodal activity (Fig. 11). Much of the PN activity during CS2 occurred toward the end of the CS period. This pattern of PN activity differed substantially from the pattern of $\mathrm{PN}$ activity seen during the initial cross-modal session (compare Figs. 5, 9), indicating that the PN acquired plasticity with additional training with CS2.

Both the IPN and PN showed more neurons that had greater activity during trials in which a CR occurred compared with trials without CRs during CS1/CS training (Figs. 10, 11). This CR-related activity was stronger in the IPN and PN during CS1/CS2 training relative to initial cross-modal training (compare Figs. 6, 11). Neurons in the PN showed a particularly striking increase in CR-related activity for CS2-US trials during CS1/CS2 training (Figs. 10, 11).

The results from CS1/CS2 training indicate that additional training with the CS2 further facilitated behavioral cross-modal transfer and learning-related activity in the IPN and PN. Neurons in the IPN showed an enhancement in CR-related activity on CS2-US trials during CS1/CS2 training relative to initial crossmodal training. The PN, which showed very little CR-related activity on CS2-US trials during cross-modal training, showed robust CR-related activity on CS2-US trials during CS1/CS2 training. Thus, the IPN showed earlier developing CR-related activity than the PN during acquisition of conditioning to the CS2 across training sessions. Additional CS1/CS2 training may have resulted in PN CR-related activity that was as robust as the CR-related activity in the IPN.

\section{Discussion}

The present experiment demonstrated that plasticity in the IPN and PN correlates with cross-modal transfer of eyeblink conditioning. Low percentages of behavioral eyeblink responses were elicited by the tone and light CSs during preexposure and unpaired training sessions. Stimulus-elicited neuronal responses were evident in both IPN and PN neurons during these sessions. During unpaired training, the majority of the neurons were responsive to either the tone or light CS; a smaller proportion of units responded to both CSs. The behavioral and neuronal responses changed after rats were given paired CS-US training in the subsequent sessions. Eyeblink CRs were acquired during CS1 acquisition, acquired again during cross-modal transfer training with CS2, and were further increased during CS1/CS2 training. Learning-related changes in neuronal firing to both CS modalities were evident among IPN and PN neurons as CRs were acquired. Learning-related changes in neuronal activity within the IPN and PN co-occurred with the CR, in which increased activity was higher on trials in which a CR occurred. The CR-related activity in the IPN and PN in the present study is consistent with findings from previous studies that used a single CS or discrimination paradigms (McCormick and Thompson, 1984; Clark et al., 1997; Bao et al., 2000; Choi and Moore, 2003; Halverson et al., 2010). IPN neurons showed learning-related activity on CS2-US trials during initial cross-modal training. In contrast, $\mathrm{PN}$ neurons did not show robust learning-related activity during CS2-US trials until CS1/CS2 training. 
A

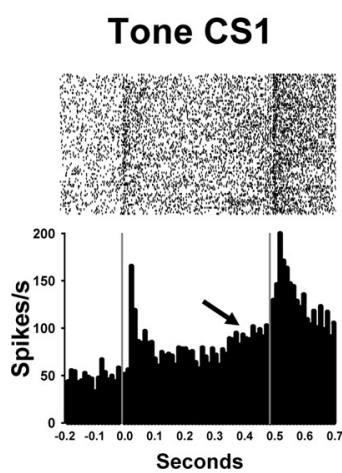

\section{Tone CS1}

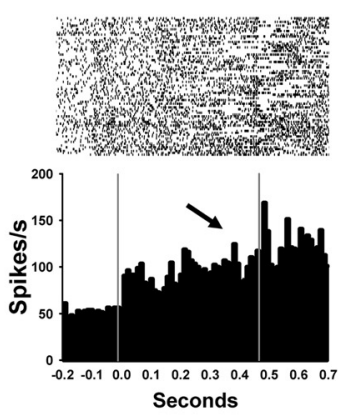

B

\section{Interpositus}
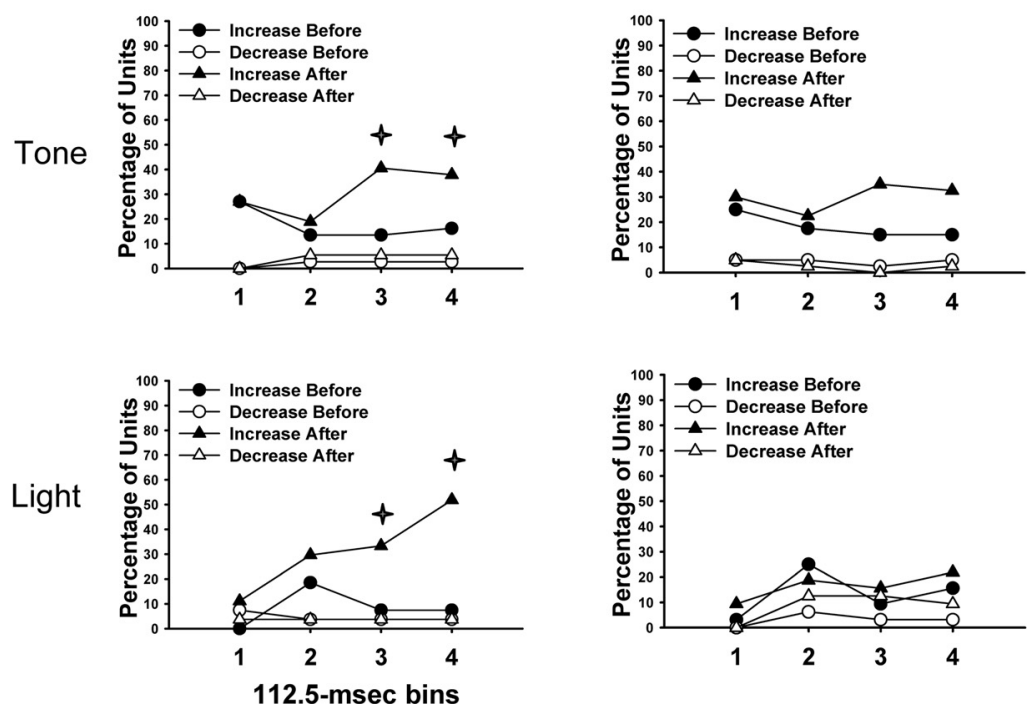

Figure 8. A, Histograms and raster plots of single-unit activity in the anterior interpositus nucleus (top) and pontine nucleus (bottom) before and after behavioral acquisition of cross-modal learning during initial cross-modal training. Arrows point to the peak of CR-related activity. Clear increases in learning-related activity do not appear on CS2-US trials before cross-modal learning occurred (middle column) but are evident in interpositus neurons after cross-modal learning (right column). The first gray line represents the onset of the $C S$, and the second represents onset of the US. B, Percentage of units in the anterior interpositus nucleus (left) and pontine nucleus (right) that exhibited significantincreases (black plots) or decreases (white plots) in activity for each $112.5 \mathrm{~ms}$ bin (1-4) of the conditioned stimuli (tone and light) before and after cross-modal learning. Four-point stars indicate significant differences between percentages before and after cross-modal training.

Neurons in the IPN and PN recorded during preexposure and unpaired sessions that responded to the CSs before paired CS-US training were primarily unimodal, i.e., they showed changes in activity to the tone or light but not to both stimuli. A greater percentage of neurons showing stimuluselicited activity was found for the tone CS during unpaired training relative to the preexposure session, although no differences were found in eyelid responses. However, no changes in behavioral or neuronal responses were detected to the light CS when comparing the preexposure and unpaired sessions. Thus, introducing a shock US to the training context during unpaired training produced a specific effect on tone-elicited activity within the IPN and PN. It is possible that enhanced neuronal responding occurred to the tone CS during unpaired training because conditioned fear was acquired to the training context. The amygdala may be involved in producing the sensory enhancement effect that was found during this phase through projections to auditory nuclei or the PN. Previous experiments have shown that bilateral lesions of the amygdala impair the rate of eyeblink conditioning with a tone CS (Weisz et al., 1992; Lee and Kim, 2004). It is possible that amygdala lesions reduce the rate of eyeblink conditioning because they prevent enhancement of sensory responding of $\mathrm{PN}$ neurons that occurs to a tone CS when it is presented in a fearful context. It is not clear why an enhancement in sensory activity was not found to the light CS during unpaired training, but one possibility is that the amygdala does not have sufficient projections to visual pontine neurons or to visual nuclei that project to the PN.

Subsequent training with paired CS1-US trials resulted in rapid acquisition of CRs and CR-related activity (i.e., greater activity on trials with CRs) within the IPN and PN. During the first day of cross-modal training, the activity of neurons in the IPN and PN was recorded as the rats were given CS1-US trials, followed immediately by CS2-US trials. This procedure resulted in acquisition of moderate levels of CRs and a corresponding moderate level of CR-related activity among IPN neurons during CS2-US trials (Figs. 5, 8). Neurons in the PN showed very little CR-related activity during the initial CS2-US training. Analysis of the emergence of CR-related activity within the CS2-US part of the initial cross-modal training session revealed that some of the IPN neurons acquired CR-related activity as CRs were acquired during initial CS2-US training. Very few of the PN neurons showed increased CR-related activity within this phase of training. Much more substantial CR-related neuronal activity was observed in both the IPN and PN on CS2-US trials with additional cross-modal learning during the subsequent CS1/CS2 training (Figs. 9-11). In- 


\section{Interpositus}

Tone CS1

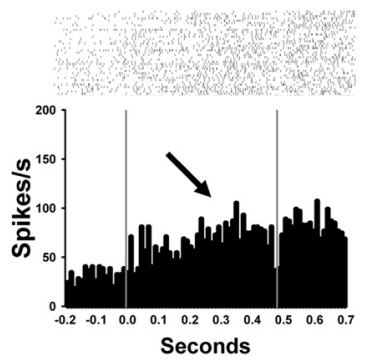

Light CS1

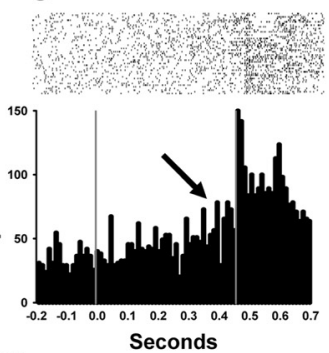

Pontine

Tone CS1

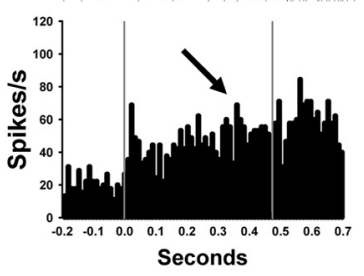

Light CS1

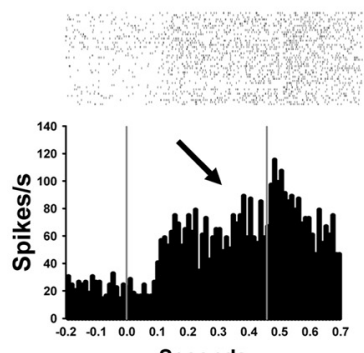

Seconds

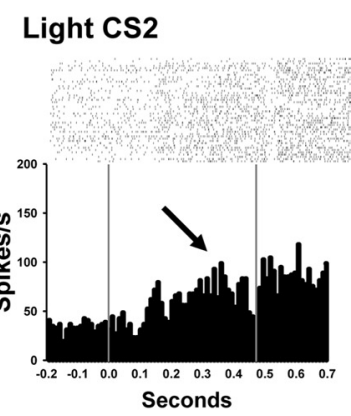

Tone CS2

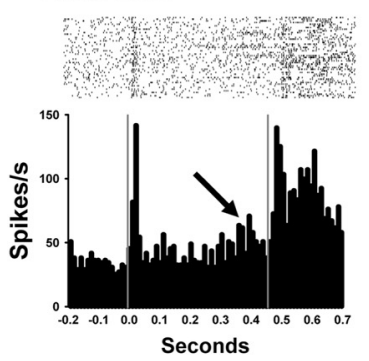

Light CS2

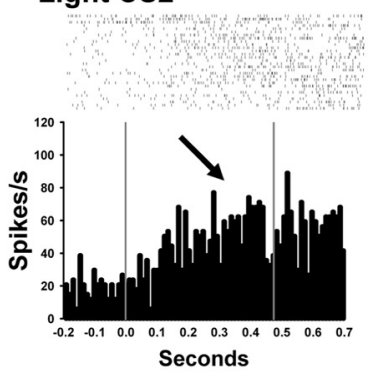

Tone CS2

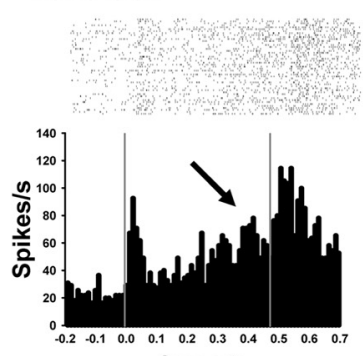

Seconds
Figure 9. Histograms and raster plots of single-unit activity in the anterior interpositus nucleus (top) and pontine nucleus (bottom) during presentations of the tone and light CSs in CS1/CS2 training. Arrows point to the peak of CR-related activity. The first gray line represents the onset of the CS, and the second represents onset of the US. Note that, with additional training, neurons in the interpositus and pontine nuclei show greater activity during the CS2.

creased IPN and PN CR-related neuronal activity corresponded with an increase in CRs during CS2-US trials during CS1/CS2 training. However, the IPN still showed stronger CR-related activity than the PN during CS1/CS2 training. These findings suggest that the primary mechanism underlying behavioral cross-modal learning is the establishment of increases in activity within the IPN during CS2-US trials that drive production of CRs.

PN neurons also established learning-related activity during cross-modal learning, but much of that learning-related activity emerged after cerebellar activity changed. Previous studies demonstrated that learning-related activity in PN can be abolished when the IPN is inactivated (Clark et al., 1997; Bao et al., 2000), suggesting that learning-related activity in the PN may be driven by the cerebellum (Clark et al., 1997). The present experiment showed that learning-related changes in activity develop more slowly during cross-modal transfer in the PN than in the IPN, which supports the hypothesis that cross-modal transfer may be driven initially by cerebellar plasticity. This hypothesis is further supported by a recent study that demonstrated abolition of crossmodal transfer after cerebellar inactivation (Campolattaro and Freeman, 2009). The cerebellum may drive the development of neuronal cross-modal transfer in the PN through excitatory feedback. Learning-related changes in PN activity may in turn facilitate acquisition of CRs during CS2-US trials by enhancing excitatory input to the cerebellum.

Tracy et al. (2001) first found evidence of multimodal processing in IPN neurons in eyeblink conditioning with rabbits. In that study, two groups of rabbits were trained with different CSs (tone and light) that were paired with a US. One group was given training with a tone/light compound CS, and the other group was given training with the individual stimuli of the compound CS. After training, IPN neuronal responses to presentations of the three different CSs types were examined. Training with the compound CS resulted in more instances of multimodal responding in IPN neurons than training with individual CS stimuli. Although the overall percentages of neurons responding to both stimulus modalities differed between the groups, a general finding was that a subset of the IPN neurons was responsive to more than one stimulus modality. The Tracy et al. (2001) study also demonstrated that training conditions can influence the degree to which IPN neurons are sensitive to different CS modalities. The amount of multimodal processing (CR-related and sensory) found in the present study with rats was greater than observed in the Tracy et al. (2001) study. Although the conditions for establishing cross-modal transfer among IPN neurons differed between studies, the mechanisms are probably the same. As proposed by Tracy et al. (2001), the threshold for firing in IPN neurons that respond to one modality may change as a result of cross-modal training by enhancing the efficacy of inputs from other sensory modalities that are normally weak (Tracy et al., 2001). It is possible that this hypothesized change in firing threshold differs between rats and rabbits or between sequential and concurrent tone/light training.

Previous eyeblink conditioning studies have shown evidence of anatomical segregation of tone and light CSs. Specifically, lesions in the dorsal lateral $\mathrm{PN}$ in rabbits have been shown to prevent conditioning with an auditory CS but not a visual CS (Steinmetz et al., 1987). A more recent study with rats found that inactivation of the lateral PN completely prevented retention of eyeblink conditioning to a tone CS but only partially impaired retention to a light CS (Halverson and Freeman, 2010a). Halverson and Freeman (2010b) also showed that inactivation of the medial PN completely prevented retention of conditioning to a light CS but only partially impaired CR retention with a tone CS. A conclusion from the Halverson and Freeman (2010b) study is that populations of neurons for auditory and visual cerebellar learning partially overlap within the PN. The present experiment showed that unimodal and multimodal processing occurs in both the lateral and medial PN, which is consistent with the hypothesis that modality-specific processing is not strictly segregated within the PN.

In summary, the present experiment used tetrode recordings to examine neuronal correlates of cross-modal transfer of eyeblink conditioning in rats. Neurons in the cerebellar interpositus 


\section{Interpositus}

Tone CS1

CR

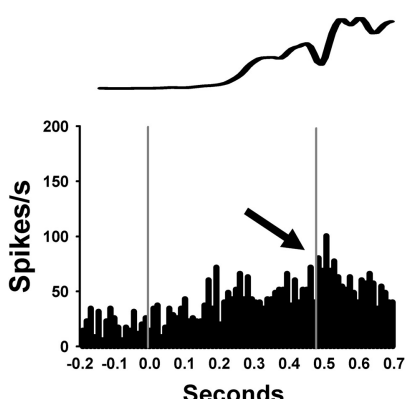

Seconds

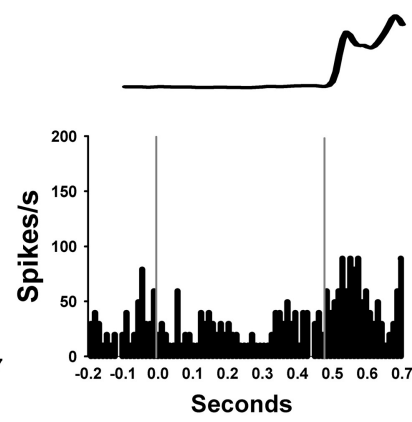

Light CS2

CR
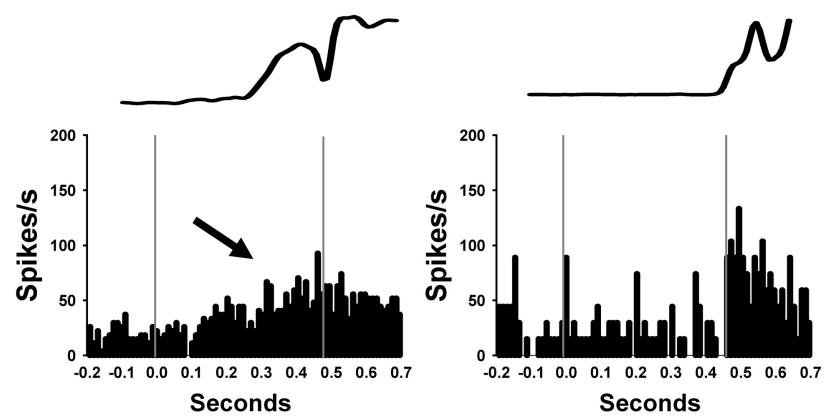

No CR

\section{Pontine}

\section{Tone CS1}

CR

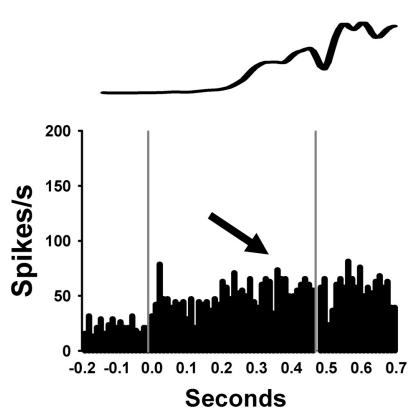

No CR

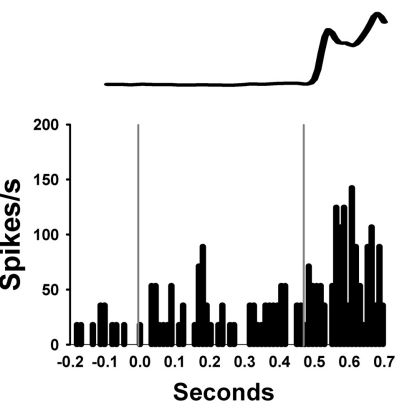

Light CS2

CR

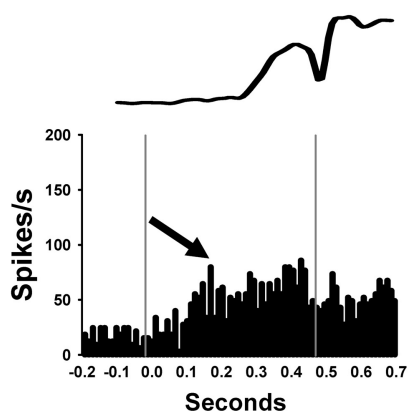

Figure 10. Eyeblink and single-unit activity in the anterior interpositus nucleus (top) and pontine nucleus (bottom) during CS1/CS2 training. Eyelid EMG and unit activity are shown for CR and no-CR trials. The first gray line represents the onset of the CS, and the second gray line represents the onset of the US. Arrows point to the peak of CR-related activity. Unit activity during CR trials shows increases in activity before onset of the US.

nucleus showed learning-related changes in activity that correlated with the development of behavioral cross-modal learning across training sessions. Pontine neurons also showed learningrelated changes in activity during cross-modal training, but these changes emerged after the development of learning-related activity in the IPN, suggesting that excitatory feedback from the IPN may have driven changes in PN activity. Excitatory feedback from the IPN to the PN may in turn facilitate the rate and magnitude of cross-modal learning through a positive feedback loop back to the cerebellum. A similar mechanism has been proposed for feedback from the cerebellum to the auditory thalamus (Halverson et al., 2010). The cerebellum may thereby provide excitatory feedback at multiple points in its CS input pathways to facilitate learning.

\section{References}

Bao S, Chen L, Thompson RF (2000) Learning- and cerebellumdependent neuronal activity in the lateral pontine nucleus. Behav Neurosci 114:254-261.

Campolattaro MM, Freeman JH (2009) Cerebellar inactivation impairs cross modal savings of eyeblink conditioning. Behav Neurosci 123: 292-302.

Cartford MC, Gohl EB, Singson M, Lavond DG (1997) The effects of reversible inactivation of the red nucleus on learning-related and auditory- evoked unit activity in the pontine nuclei of classically conditioned rabbits. Learn Mem 3:519-531.

Choi JS, Moore JW (2003) Cerebellar neuronal activity expresses the complex topography of conditioned eyeblink responses. Behav Neurosci 117:1211-1219.

Clark RE, Gohl EB, Lavond DG (1997) The learning-related activity that develops in the pontine nuclei during classical eye-blink conditioning is dependent on the interpositus nucleus. Learn Mem 3:532-544.

Freeman JH Jr, Muckler AS (2003) Developmental changes in eyeblink conditioning and neuronal activity in the pontine nuclei. Learn Mem 10:337-345.

Freeman JH Jr, Nicholson DA (1999) Neuronal activity in the cerebellar interpositus and lateral pontine nuclei during inhibitory classical conditioning of the eyeblink response. Brain Res 833:225-233.

Freeman JH Jr, Nicholson DA (2000) Developmental changes in eye-blink conditioning and neural activity in the cerebellar interpositus nucleus. J Neurosci 20:813-819.

Halverson HE, Freeman JH (2010a) Medial auditory thalamic input to the lateral pontine nuclei is necessary for auditory eyeblink conditioning. Neurobiol Learn Mem 93:92-98.

Halverson HE, Freeman JH (2010b) Ventral lateral geniculate input to the medial pons is necessary for visual eyeblink conditioning in rats. Learn Mem 17:80-85.

Halverson HE, Lee I, Freeman JH (2010) Associative plasticity in the medial auditory thalamus and cerebellar interpositus nucleus during eyeblink conditioning. J Neurosci 30:8787-8796. 


\section{A Interpositus}

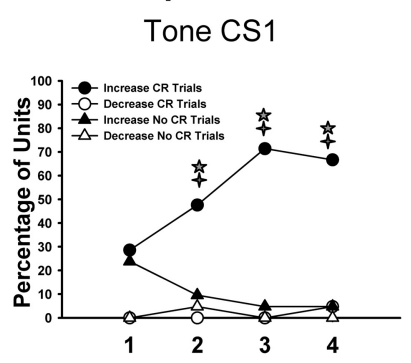

Light CS1

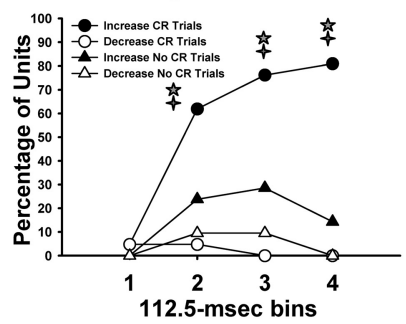

B Pontine

Tone CS1

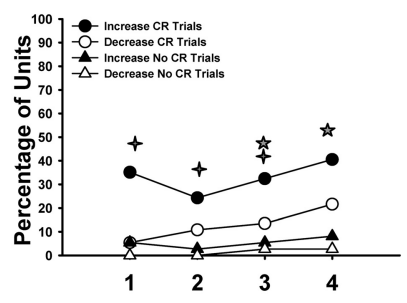

Light CS1

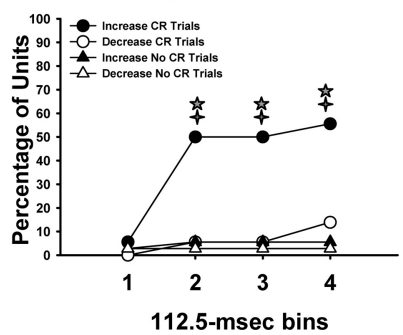

112.5-msec bins

Figure 11. Percentage of units in the anterior interpositus nucleus $(\boldsymbol{A})$ and pontine nucleus $(\boldsymbol{B})$ that exhibited significant increases (black plots) or decreases (white plots) in activity for each $112.5 \mathrm{~ms}$ bin (1-4) of the conditioned stimuli during CS1/CS2 training divided into CR (circles) and non-CR (triangles) trial types. Four-point stars indicate significant differences in bin percentage between the $C R$ and non-CR trial types. Five-point stars indicate significant differences in bin percentage between paired and unpaired training (see Fig. 4B).
Hesslow G, Svensson P, Ivarsson M (1999) Learned movements elicited by direct stimulation of cerebellar mossy fiber afferents. Neuron 24:179-185.

Holt PE, Kehoe EJ (1985) Cross-modal transfer as a function of similarities between training tasks in classical conditioning of the rabbit. Anim Learn Behav 13:51-59.

Kehoe EJ, Morrow LD, Holt PE (1984) General transfer across sensory modalities survives reductions in the original conditioned reflex in the rabbit. Anim Learn Behav 12:129-136.

Lee T, Kim JJ (2004) Differential effects of cerebellar, amygdalar, and hippocampal lesions on classical eyeblink conditioning in rats. J Neurosci 24:3242-3250.

Lewis JL, Lo Turco JJ, Solomon PR (1987) Lesions of the middle cerebellar peduncle disrupt acquisition and retention of the rabbit's classically conditioned nictitating membrane response. Behav Neurosci 101:151-157.

Mauk MD, Donegan NH (1997) A model of pavlovian eyelid conditioning based on the synaptic organization of the cerebellum. Learn Mem 4:130-158.

McCormick DA, Thompson RF (1984) Neuronal responses of the rabbit cerebellum during acquisition and performance of a classically conditioned nictitating membrane-eyelid response. J Neurosci 4:2811-2822.

McCormick DA, Lavond DG, Thompson RF (1983) Neuronal responses of the rabbit brainstem during performance of the classically conditioned nictitating membrane (NM)/eyelid response. Brain Res 271:73-88.

Nahinsky ID, Lucas BA, Edgell SE, Overfelt J, Loeb R (2004) How learning one category influences the learning of another: intercategory generalization based on analogy and specific stimulus information. Am J Psychol 117:317-340.

Nicholson DA, Freeman JH Jr (2000) Developmental changes in eye-blink conditioning and neuronal activity in the inferior olive. J Neurosci 20:8218-8226.

Nicholson DA, Freeman JH Jr (2002) Neuronal correlates of conditioned inhibition of the eyeblink response in the anterior interpositus nucleus. Behav Neurosci 116:22-36.

Nicholson DA, Freeman JH Jr (2003) Addition of inhibition in the olivocerebellar system and the ontogeny of a motor memory. Nat Neurosci 6:532-537.

Steinmetz JE, Rosen DJ, Woodruff-Pak DS, Lavond DG, Thompson RF (1986) Rapid transfer of training occurs when direct mossy fiber stimulation is used as a conditioned stimulus for classical eyelid conditioning. Neurosci Res 3:606-616.

Steinmetz JE, Logan CG, Rosen DJ, Thompson JK, Lavond DG, Thompson RF (1987) Initial localization of the acoustic conditioned stimulus projection system to the cerebellum essential for classical eyelid conditioning. Proc Natl Acad Sci U S A 84:3531-3535.

Steinmetz JE, Lavond DG, Thompson RF (1989) Classical conditioning in rabbits using pontine nucleus stimulation as a conditioned stimulus and inferior olive stimulation as an unconditioned stimulus. Synapse 3:225-233.

Thompson RF, Steinmetz JE (2009) The role of the cerebellum in classical conditioning of discrete behavioral responses. Neuroscience 162:732-755.

Tracy JA, Britton GB, Steinmetz JE (2001) Comparison of single unit responses to tone, light, and compound conditioned stimuli during rabbit classical eyeblink conditioning. Neurobiol Learn Mem 76:253-267.

Weisz DJ, Harden DG, Xiang Z (1992) Effects of amygdala lesions on reflex facilitation and conditioned response acquisition during nictitating membrane response conditioning in rabbit. Behav Neurosci 106:262-273. 